\title{
A NOTE ON POSITIVITY OF THE CM LINE BUNDLE
}

\author{
J. FINE AND J. ROSS
}

\begin{abstract}
We show that positivity of the CM line associated to a family of polarised varieties is intimately related to the stability of its members. We prove that the CM line is nef on any curve which meets the stable locus, and that it is pseudoeffective (i.e. in the closure of the effective cone) as long as there is at least one stable fibre. We give examples showing that the CM line can be strictly negative or strictly positive on curves in the unstable locus.
\end{abstract}

\section{INTRODUCTION}

A famous conjecture of Yau relates the existence of Kähler metrics of constant scalar curvature to stability in Geometric Invariant Theory (GIT). It is thought that this stability notion should be with respect to the CM line bundle on the Hilbert scheme, originally defined by Tian [14. Unfortunately, it is not clear directly from the definition when this line bundle is positive (e.g. ample), so one cannot define stability in terms of the existence of non-vanishing invariant sections as is standard in GIT. Instead, the definition of stability that is commonplace is made by analogy with the Hilbert-Mumford criterion, and requires that for all one parameter subgroups the the Hilbert-Mumford weight function has the favourable sign.

We start this note by defining the CM line following Paul-Tian 12 and then show how it arises as the leading order term in an expansion of some naturally defined line bundles. We then turn to positivity properties of the CM line (we use the term positivity loosely here to mean ample, nef, effective and so on). Roughly speaking, we show that the CM line is non-negative on the "stable" locus (more precisely, the asymptotically Hilbert semistable locus) and that, as long as the stable locus is non-empty, the CM line is pseudoeffective. We end with examples showing that outside the stable locus the CM line is badly behaved: there are examples of families of smooth unstable surfaces for which the CM line is strictly negative, and other such examples for which it is positive.

Notation and Conventions: If $\mathcal{L}$ is a line bundle its powers are $\mathcal{L}^{k}=\mathcal{L}^{\otimes k}$ and $\mathcal{L}^{-k}=\left(\mathcal{L}^{*}\right)^{\otimes k}$. If $\mathcal{X} \rightarrow B$ is a family of schemes the fibre over a point $b \in B$ is $\mathcal{X}_{b}$ and if $\mathcal{L} \rightarrow \mathcal{X}$ is a line bundle then $\mathcal{L}_{b}=\left.\mathcal{L}\right|_{\mathcal{X}_{b}}$. By a polarised variety or scheme $(X, L)$ we mean a choice of ample line bundle $L$ on $X$.

We say that a line bundle on $B$ is nef if it has non-negative degree on any irreducible curve $C$ in $B$. It is effective if some positive tensor power has a section, and it is pseudoeffective if its first Chern class lies on the closure of the cone of effective divisors in the Néron-Severi space $N^{1}(B)$. We work throughout over the complex numbers.

Acknowledgements: We would like to thank Sean Paul and Gang Tian for useful conversations, and explaining to us their definition of the CM line [12. 


\section{Definition of the CM Line BUndLe}

Let $\pi: \mathcal{X} \rightarrow B$ be a proper flat morphism of schemes of constant relative dimension $n \geq 1$ and let $\mathcal{L}$ be a relatively ample line bundle on $\mathcal{X}$. We will assume throughout that $B$ is irreducible, and that $\mathcal{X}$ has pure dimension.

The CM line is defined in terms of the determinant of the pushdown of $\mathcal{L}^{k}$ given by

$$
\operatorname{det} \pi_{!}\left(\mathcal{L}^{k}\right)=\operatorname{det} R^{\bullet} \pi\left(\mathcal{L}^{k}\right)=\bigotimes_{i}\left(\operatorname{det} R^{i} \pi_{*}\left(\mathcal{L}^{k}\right)\right)^{(-1)^{i}}
$$

As $\mathcal{L}$ is relatively ample the terms $R^{i} \pi_{*}\left(\mathcal{L}^{k}\right)$ vanish for $i>0$ and $k \gg 0$ so $\operatorname{det} \pi_{!}\left(\mathcal{L}^{k}\right)=\operatorname{det} \pi_{*}\left(\mathcal{L}^{k}\right)$. We will rely on the fact that $\operatorname{det} \pi_{!}\left(\mathcal{L}^{k}\right)$ has a polynomial expansion in terms of some fixed line bundles $\lambda_{i}$ on $B$.

Theorem 2.1 (Mumford-Knudsen). There exist line bundles $\lambda_{i}=\lambda_{i}(\mathcal{X}, \mathcal{L})$ on $B$ such that for all $k$

$$
\operatorname{det} \pi_{!}\left(\mathcal{L}^{k}\right) \cong \lambda_{n+1}^{\left(\begin{array}{c}
k \\
n+1
\end{array}\right)} \otimes \lambda_{n}^{\left(\begin{array}{c}
k \\
n
\end{array}\right)} \otimes \cdots \otimes \lambda_{0}
$$

Proof. See (GIT 11] page 230) or (Theorem 4 [10]).

We now come to the definition of the CM line. Although it is not immediately apparent, it turns out that this is a very natural line bundle to consider (see Section 3). Write the Hilbert polynomial of the fibres of $\mathcal{X}$ as

$$
p(k)=\chi\left(\mathcal{L}_{b}^{k}\right)=a_{0} k^{n}+a_{1} k^{n-1}+O\left(k^{n-2}\right),
$$

which by flatness of the family is independent of $b \in B$. As $\mathcal{L}_{b}$ is ample the term $a_{0}$ is strictly positive, so we can set

$$
\mu=\mu(\mathcal{X}, \mathcal{L})=\frac{2 a_{1}}{a_{0}}
$$

Definition 2.3. (Paul-Tian [12]) The CM line bundle associated to the family $(\mathcal{X}, \mathcal{L})$ is

$$
\lambda_{\mathrm{CM}}=\lambda_{\mathrm{CM}}(\mathcal{X}, \mathcal{L})=\lambda_{n+1}^{\mu+n(n+1)} \otimes \lambda_{n}^{-2(n+1)} .
$$

Paul-Tian show in 12 that $\lambda_{\mathrm{CM}}$ agrees with Tian's original definition of the CM line in terms of the pushdown of a certain virtual bundle on $\mathcal{X}$ [14. For example, suppose $\pi: \mathcal{X} \rightarrow B$ has a relative canonical bundle $K_{\mathcal{X} / B}$. An easy calculation with the Grothendieck-Riemann-Roch formula applied to $\mathcal{L}^{k}$ gives

$$
\begin{aligned}
c_{1}\left(\lambda_{n+1}\right) & =\pi_{*} c_{1}(\mathcal{L})^{n+1} \\
n c_{1}\left(\lambda_{n+1}\right)-2 c_{1}\left(\lambda_{n}\right) & =\pi_{*}\left(c_{1}(\mathcal{L})^{n} c_{1}\left(K_{\mathcal{X} / B}\right)\right) .
\end{aligned}
$$

Hence

$$
c_{1}\left(\lambda_{\mathrm{CM}}\right)=\pi_{*}\left[\mu c_{1}(\mathcal{L})^{n+1}+(n+1) c_{1}\left(K_{\mathcal{X} / B}\right) c_{1}(\mathcal{L})^{n}\right]
$$

The definition of the CM line is made so that the Hilbert-Mumford weight function (in the sense of geometric invariant theory) equals Donaldson's version of the Futaki invariant [5] as in the following lemma. This is the reason for the link between $\lambda_{\mathrm{CM}}$ and the problem of finding Kähler metrics of constant scalar curvature. We refer the interested reader to 15 for an introduction to this topic. 
Lemma 2.5 (Paul-Tian). Suppose that $\mathbb{C}^{\times}$acts on $(\mathcal{X}, \mathcal{L})$ covering an action on $B$, and that $b \in B$ is a fixed point. Write the weight (i.e. the sum of the eigenvalues) of the induced action on $H^{0}\left(\mathcal{X}_{b}, \mathcal{L}_{b}^{k}\right)$ as a polynomial $w(k)$ with coefficients

$$
w(k)=b_{0} k^{n+1}+b_{1} k^{n}+O\left(k^{n-1}\right) .
$$

Then the weight of the induced action on $\left.\lambda_{\mathrm{CM}}\right|_{b}$ is the Futaki invariant

$$
F_{1}=\frac{2(n+1) !}{a_{0}}\left(b_{1} a_{0}-b_{0} a_{1}\right) .
$$

Remark 2.6. The CM line is homogeneous of order $n$, i.e. if $r \in \mathbb{N}$ then

$$
\lambda_{\mathrm{CM}}\left(\mathcal{X}, \mathcal{L}^{r}\right)=r^{n} \lambda_{\mathrm{CM}}(\mathcal{X}, \mathcal{L}) .
$$

Thus by clearing denominators we can define $\lambda_{\mathrm{CM}}(\mathcal{X}, \mathcal{L})$ as a $\mathbb{Q}$-line bundle when $\mathcal{L}=\mathcal{O}(D)$ for some relatively ample $\mathbb{Q}$-divisor $D$, and it makes sense to talk about $\lambda_{\mathrm{CM}}$ being positive (e.g. ample, nef or pseudoeffective) in this case.

\section{The CM Line AS A LEADing ORDER TERM}

We now give an alternative description of the CM line in terms of some naturally defined line bundles. We retain the notation from the previous section, so $\pi: \mathcal{X} \rightarrow B$ is a proper flat family of schemes of relative dimension $n, \mathcal{L} \rightarrow \mathcal{X}$ is a relatively ample line bundle on $\mathcal{X}$ and the fibres of $\mathcal{X}$ have Hilbert polynomial $p$. We set

$$
\lambda(k)=\operatorname{det} \pi_{!}\left(\mathcal{L}^{k}\right) .
$$

Suppose that $\sigma$ is a line bundle on $B$. If for $k \gg 0$ we replace $\mathcal{L}$ by $\mathcal{L} \otimes \pi^{*} \sigma$ then by the projection formula $\lambda(k)$ becomes

$$
\operatorname{det} \pi_{!}\left(\left(\mathcal{L} \otimes \pi^{*} \sigma\right)^{k}\right)=\operatorname{det}\left(\pi_{*}\left(\mathcal{L}^{k}\right) \otimes \sigma^{k}\right)=\operatorname{det} \pi_{*}\left(\mathcal{L}^{k}\right) \otimes \sigma^{k p(k)} .
$$

Thus for $k \gg 0$ the line bundle

$$
\lambda_{\mathrm{Hilb}}(\mathcal{X}, \mathcal{L}, k)=\lambda(k)^{p(1)} \otimes \lambda(1)^{-k p(k)}
$$

is rigid, i.e. independent of twisting $\mathcal{L}$ by $\pi^{*} \sigma$, and thus should be thought of as a natural line bundle associated purely to the family of polarised varieties $\left(\mathcal{X}_{b}, \mathcal{L}_{b}\right)$.

To relate $\lambda_{\text {Hilb }}$ to $\lambda_{\mathrm{CM}}$ we replace $\mathcal{L}$ by $\mathcal{L}^{r}$ and consider

$$
\lambda_{\mathrm{Hilb}}\left(\mathcal{X}, \mathcal{L}^{r}, k\right)=\lambda(k r)^{r p(r)} \otimes \lambda(r)^{-k r p(k r)}
$$

which now depends on two variables $k$ and $r$. The CM line is the leading order term of this line bundle for $k \gg r \gg 0$ as in the following proposition.

Proposition 3.1. Let $\mathcal{X} \rightarrow B$ and $\mathcal{L}$ be as above. Then

$$
\lambda_{\mathrm{Hilb}}\left(\mathcal{X}, \mathcal{L}^{r}, k\right)=\lambda_{\mathrm{CM}}(\mathcal{X}, \mathcal{L})^{\frac{a_{0}}{2(n+1) !} k^{n+1} r^{2 n}} \otimes \epsilon(r, k)
$$

where $\epsilon(r, k)$ consists of "lower order terms" in $k$ and $r$. More precisely there exist fixed line bundles $\epsilon_{i}$ on $B$ such that

$$
\epsilon(r, k)=\bigotimes_{i, j} \epsilon^{k^{i} r^{j}}
$$

where the product is over $i=0, \ldots, n+1$ and $j=0, \ldots, 2 n+1$ with $j \leq 2 n-1$ if $i=n+1$. 
Proof. Recall from (2.1) that there are line bundles $\lambda_{i}$ on $B$ and an expansion

$$
\operatorname{det} \pi_{!}\left(\mathcal{L}^{k}\right) \cong \lambda_{n+1}^{\left(\begin{array}{c}
k \\
n+1
\end{array}\right)} \otimes \lambda_{n}^{\left(\begin{array}{c}
k \\
n
\end{array}\right)} \otimes \cdots \otimes \lambda_{0} .
$$

For convenience, define the Cornalba-Harris line associated to $(\mathcal{X}, \mathcal{L})$ as

$$
\lambda_{\mathrm{CH}}=\lambda_{\mathrm{CH}}(\mathcal{X}, \mathcal{L})=\lambda_{n+1}^{p(1)} \otimes \lambda(1)^{-a_{0}(n+1) !} .
$$

This is the leading order piece of $\lambda_{\mathrm{Hilb}}(\mathcal{X}, \mathcal{L}, k)$ as $k \gg 0$ since, omitting terms of order $k^{n}$, we have

$$
\begin{aligned}
\lambda_{\mathrm{Hilb}}(\mathcal{X}, \mathcal{L}, k) & =\lambda(k)^{p(1)} \otimes \lambda(1)^{-k p(k)} \\
& =\lambda_{n+1}^{\left(\begin{array}{c}
k \\
n+1
\end{array}\right) p(1)} \otimes \cdots \otimes \lambda(1)^{-k\left(a_{0} k^{n}+\cdots\right)} \\
& =\left(\lambda_{n+1}^{k^{n+1} p(1)} \otimes \lambda(1)^{-a_{0}(n+1) ! k^{n+1}}\right)^{\frac{1}{(n+1) !}} \otimes \cdots \\
& =\lambda_{\mathrm{CH}}(\mathcal{X}, \mathcal{L})^{\frac{k^{n+1}}{(n+1) !}} \otimes \cdots
\end{aligned}
$$

Replacing $\mathcal{L}$ by $\mathcal{L}^{r}$ this yields

$$
\lambda_{\mathrm{Hilb}}\left(\mathcal{X}, \mathcal{L}^{r}, k\right)=\lambda_{\mathrm{CH}}\left(\mathcal{X}, \mathcal{L}^{r}\right)^{\frac{k^{n+1}}{(n+1) !}} \otimes \cdots .
$$

Thus we need to expand $\lambda_{\mathrm{CH}}\left(\mathcal{X}, \mathcal{L}^{r}\right)$ as a function of $r$. To this end notice that replacing $\mathcal{L}$ by $\mathcal{L}^{r}$ has the effect of replacing $\lambda_{n+1}$ by $\lambda_{n+1}^{r^{n+1}}$ and $a_{0}$ by $a_{0} r^{n}$. So for $r \geq 1$ we have

$$
\lambda_{\mathrm{CH}}\left(\mathcal{X}, \mathcal{L}^{r}\right)=\lambda_{n+1}^{p(r) r^{n+1}} \otimes \lambda(r)^{-r^{n} a_{0}(n+1) !} .
$$

Now $\left(\begin{array}{c}r \\ n+1\end{array}\right)=\frac{r^{n+1}}{(n+1) !}-\frac{n(n+1)}{2(n+1) !} r^{n}+O\left(r^{n-1}\right)$, so up to terms of order $r^{n-1}$,

$$
\lambda(r)=\lambda_{n+1}^{\left(\begin{array}{c}
r \\
n+1
\end{array}\right)} \otimes \lambda_{n}^{\left(\begin{array}{c}
r \\
n
\end{array}\right)} \otimes \cdots=\lambda_{n+1}^{\frac{r^{n+1}}{(n+1) !}} \otimes \lambda_{n+1}^{-\frac{n(n+1)}{2(n+1) !} r^{n}} \otimes \lambda_{n}^{\frac{r^{n}}{n !}} \otimes \cdots
$$

Thus, up to terms of order $r^{2 n-1}$,

$$
\begin{aligned}
\lambda_{\mathrm{CH}}\left(\mathcal{X}, \mathcal{L}^{r}\right) & =\lambda_{n+1}^{p(r) r^{n+1}-a_{0} r^{2 n+1}} \otimes \lambda_{n+1}^{\frac{n(n+1)}{2} a_{0} r^{2 n}} \otimes \lambda_{n}^{-a_{0}(n+1) r^{2 n}} \otimes \cdots \\
& =\lambda_{n+1}^{\left(a_{1}+\frac{a_{0} n(n+1)}{2}\right) r^{2 n}} \otimes \lambda_{n}^{-a_{0}(n+1) r^{2 n}} \otimes \cdots \\
& =\left(\lambda_{n+1}^{\mu+n(n+1)} \lambda_{n}^{-2(n+1)}\right)^{\frac{a_{0}}{2} r^{2 n}} \otimes \cdots \\
& =\lambda_{\mathrm{CM}}(\mathcal{X}, \mathcal{L})^{\frac{a_{0}}{2} r^{2 n}} \otimes \cdots
\end{aligned}
$$

where in the second line we have use that $p(r)-a_{0} r^{n}=a_{1} r^{n-1}+O\left(r^{n-2}\right)$. Thus the Proposition follows from this and (3.4). Clearly from the above calculation the line bundle $\epsilon(r, k)$ is of the form claimed.

Remark 3.5. It is convenient to define a $\mathbb{Q}$-line bundle by

$$
\lambda_{\mathrm{CM}}^{\prime}(\mathcal{X}, \mathcal{L})=\lambda_{\mathrm{CM}}(\mathcal{X}, \mathcal{L})^{\frac{1}{2 a_{0}(n+1) !}},
$$

where $n$ is the relative dimension of $\mathcal{X}$ and $a_{0}$ is the leading order term of the Hilbert polynomial of the fibres. Then the previous Proposition becomes

$$
\lambda_{\mathrm{Hilb}}\left(\mathcal{X}, \mathcal{L}^{r}, k\right)=\lambda_{\mathrm{CM}}^{\prime}(\mathcal{X}, L)^{a_{0}^{2} k^{n+1}} r^{2 n} \otimes \cdots .
$$




\section{Positivity of the CM line on the stable locus}

We now make stability assumptions on a general fibre of $\mathcal{X}$ to deduce positivity properties of $\lambda_{\mathrm{CM}}$. This idea was exploited by Cornalba-Harris to show certain divisor classes on the moduli space of curves are nef 3 . We recall the definition of Hilbert stability of a polarised scheme $(X, L)$. Suppose first that $L$ is very ample. Then, up to change of coordinates, we have an embedding of $X$ in $\mathbb{P}^{N}$. For $k \gg 0$ this yields a point $\operatorname{Hilb}(X, L, k)$ in the Hilbert $\operatorname{Scheme} \operatorname{Hilb}\left(\mathbb{P}^{N}, k\right)$ representing the subspace of degree $k$ polynomials on $\mathbb{P}^{N}$ that vanish along $X$.

Definition 4.1. We say that a polarised variety $(X, L)$ is Hilbert semistable if for arbitrarily large $k$ the point $\operatorname{Hilb}(X, L, k)$ is semistable in the sense of GIT under the natural $S L_{N+1}$ action. (This is to be understood with respect to the hyperplane bundle on $\operatorname{Hilb}\left(\mathbb{P}^{n}, k\right)$ coming from the embedding in the Grassmannian of subspaces of degree $k$ polynomials on $\left.\mathbb{P}^{N}\right)$. We say that $(X, L)$ is asymptotically Hilbert semistable if $\left(X, L^{r}\right)$ is Hilbert semistable for arbitrarily large $r$.

Theorem 4.2 (Cornalba-Harris). Suppose that $\mathcal{L}$ is relatively very ample and without higher cohomology, and that for some $b \in B$ the fibre $\left(\mathcal{X}_{b}, \mathcal{L}_{b}\right)$ of $(\mathcal{X}, \mathcal{L})$ is Hilbert semistable. Then for arbitrarily large $k, \lambda_{\mathrm{Hilb}}(\mathcal{X}, \mathcal{L}, k)$ is effective.

Moreover there is a section of some power of $\lambda_{\mathrm{Hilb}}(\mathcal{X}, \mathcal{L}, k)$ that does not vanish at $b$. Thus if $C \subset B$ is an irreducible curve containing $b$ then for arbitrarily large $k$

$$
c_{1}\left(\lambda_{\mathrm{Hilb}}(\mathcal{X}, \mathcal{L}, k)\right) . C>0 .
$$

Sketch proof (see [3] or 9] Chapter 6D). For $k \gg 0$ there is a generically surjective map of vector bundles

$$
\alpha_{k}: S^{k} \operatorname{det} \pi_{*}(\mathcal{L}) \rightarrow \pi_{*}\left(\mathcal{L}^{k}\right)
$$

where $S^{k}$ denotes the $k$-th symmetric product. Thus we get an induced map

$$
\beta_{k}: \Lambda^{p(k)}\left(S^{k} \operatorname{det} \pi_{*}(\mathcal{L})\right) \rightarrow \Lambda^{p(k)} \pi_{*}\left(\mathcal{L}^{k}\right)=\operatorname{det} \pi_{*}\left(\mathcal{L}^{k}\right)
$$

The kernel of this map at a point $b \in B$ is the Hilbert point of the fibre $\left(\mathcal{X}_{b}, \mathcal{L}_{b}\right)$. The stability assumption means that for arbitrarily large $k$ there is an SL-invariant homogeneous polynomial $P$ that does not vanish at $\left.\beta_{k}\right|_{b}$. We may assume that $P$ has degree $p(1) m$ for some $m \geq 1$. Thus picking a trivialisation of $\pi_{*}(\mathcal{L})$ gives a regular local function $f$ on $B$ given by $f(t)=P\left(\left.\beta_{k}\right|_{t}\right)$. The fact that $P$ is SLinvariant implies that changing this choice of trivialisation by multiplication by a matrix $M$ scales $f$ by a factor $(\operatorname{det} M)^{-p(k) k / p(1)}$. Thus $f$ defines a section of

$$
\operatorname{det} \pi_{*}\left(\mathcal{L}^{k}\right)^{p(1) m} \otimes \operatorname{det}\left(\pi_{*} \mathcal{L}\right)^{-k p(k) m}=\lambda_{\mathrm{Hilb}}(\mathcal{X}, \mathcal{L}, k)^{m}
$$

that does not vanish at $b$. The rest of the theorem follows immediately.

Theorem 4.3. Assume that for at least one $b$ the fibre $\left(\mathcal{X}_{b}, \mathcal{L}_{b}\right)$ is asymptotically Hilbert semistable. Then $\lambda_{\mathrm{CM}}$ is pseudoeffective.

Moreover if $C$ is a curve in $B$ and there is $a b \in C$ such that $\left(\mathcal{X}_{b}, \mathcal{L}_{b}\right)$ is asymptotically Hilbert semistable then $c_{1}\left(\lambda_{\mathrm{CM}}\right) . C \geq 0$.

Proof. Pick $b$ so $\left(\mathcal{X}_{b}, \mathcal{L}_{b}\right)$ is asymptotically Hilbert stable. This means that for arbitrarily large $r$ the fibre $\left(\mathcal{X}_{b}, \mathcal{L}_{b}^{r}\right)$ is Hilbert semistable. By Theorem 4.2 this implies $\lambda_{\text {Hilb }}\left(\mathcal{X}, \mathcal{L}^{r}, k\right)$ is effective for arbitrarily large $k$ (and has positive degree along any irreducible curve $C$ containing $b$ ). From (3.1) we know that $\lambda_{\mathrm{CM}}(\mathcal{X}, \mathcal{L})$ is the leading order term of $\lambda_{\text {Hilb }}\left(\mathcal{X}, \mathcal{L}^{r}, k\right)$. Thus letting $k$ tend to infinity and then $r$ 
tend to infinity we deduce that $\lambda_{\mathrm{CM}}(\mathcal{X}, \mathcal{L})$ is pseudoeffective (and has non-negative degree along $C$ ).

\section{Remark 4.4.}

(1) In [3] Cornalba-Harris use analogous reasoning to show that $\lambda_{\mathrm{CH}}\left(\mathcal{X}, \mathcal{L}^{r}\right)$ defined in (3.3) is pseudoeffective. Viehweg has a similar argument in 16. to show positivity of certain line bundles connected to families of general type surfaces. Ultimately Viehweg relies on Gieseker's proof that surfaces of general type are asymptotically Hilbert stable with respect to high pluricanonical embeddings 8 .

(2) That the CM line is the leading order piece of $\lambda_{\mathrm{CH}}$ was originally observed by the first author in [6]. The difference between this and the argument given there is that we use a different definition of the CM line and so avoid any mention of a relative canonical bundle of $\mathcal{X} \rightarrow B$.

\section{Remark 4.5.}

(1) Recent work connecting stability to the existence of constant scalar curvature Kähler metrics gives conditions under which the above theorem can be applied. Suppose there is a $b \in B$ such that the fibre $\left(\mathcal{X}_{b}, \mathcal{L}_{b}\right)$ is smooth, has no infinitesimal automorphisms, and that there exists a Kähler metric on $\mathcal{X}_{b}$ which has constant scalar curvature and whose cohomology class is $c_{1}\left(\mathcal{L}_{b}\right)$. Then Donaldson shows that $\left(\mathcal{X}_{b}, \mathcal{L}_{b}\right)$ is asymptotically Hilbert semistable [4] and thus (4.3) applies. Such a metric exists, for example, when either

(a) $\mathcal{L}_{b}=K_{\mathcal{X}_{b}}$ is the canonical bundle (as then a Kähler-Einstein metric exists by results of Aubin and Yau [2, 17]); or

(b) $K_{\mathcal{X}_{b}}$ is trivial and $\mathcal{L}$ is arbitrary (by existence of a Ricci flat metric by Yau's proof of the Calabi conjecture 17]).

(2) A related result is proved by Fujiki-Schumacher 7]. Suppose that $B$ is a smooth curve, $\mathcal{X} \rightarrow B$ is a non-trivial family whose fibres are all smooth with no holomorphic vector fields and, moreover, that for each $b$ the ample class $c_{1}\left(\mathcal{L}_{b}\right)$ on $\mathcal{X}_{b}$ contains a constant scalar curvature Kähler metric. They prove that $c_{1}\left(\lambda_{\mathrm{CM}}\right) . B>0$. Note that this is neither weaker or stronger than the conclusions drawn above. On the one hand, ample implies nef, on the other hand, Theorem 4.3 makes no smoothness assumptions.

We can also use Proposition 3.1 to prove other facts about the CM line. For instance it is immediate that it is rigid, i.e. unchanged when $\mathcal{L}$ is twisted by a line pulled back from the base:

Corollary 4.6. For any line bundle $\sigma$ on $B$,

$$
\lambda_{\mathrm{CM}}\left(\mathcal{X}, \mathcal{L} \otimes \pi^{*} \sigma\right)=\lambda_{\mathrm{CM}}(\mathcal{X}, \mathcal{L})
$$

Proof. This follows from (4.3) and the fact that $\lambda_{\text {Hilb }}$ is unchanged if $\mathcal{L}$ is replaced by $\mathcal{L} \otimes \pi^{*} \sigma$.

Another application concerns the $\mathrm{CM}$ line on products. Let $\mathcal{X}_{1} \rightarrow B$ and $\mathcal{X}_{2} \rightarrow B$ be proper flat families of schemes of constant relative dimension $n$ and $m$ respectively. For $i=1,2$ let $\mathcal{L}_{i} \rightarrow \mathcal{X}_{i}$ be relatively ample line bundles. Denote by $\mathcal{X}=\mathcal{X}_{1} \times_{B} \mathcal{X}_{2} \rightarrow B$ the fibred product with projections $q_{i}: \mathcal{X} \rightarrow \mathcal{X}_{i}$, and by $\mathcal{L}=q_{1}^{*} \mathcal{L}_{1} \otimes q_{2}^{*} \mathcal{L}_{2}$ the product polarisation. 
Corollary 4.7. With notation as above and $\lambda_{\mathrm{CM}}^{\prime}$ defined as in (3.6),

$$
\lambda_{\mathrm{CM}}^{\prime}\left(\mathcal{X}_{1} \times_{B} \mathcal{X}_{2}, \mathcal{L}\right)=\lambda_{\mathrm{CM}}^{\prime}\left(\mathcal{X}_{1}, \mathcal{L}_{1}\right) \otimes \lambda_{\mathrm{CM}}^{\prime}\left(\mathcal{X}_{2}, \mathcal{L}_{2}\right)
$$

Proof. It is sufficient to prove the formula for the product $\mathcal{X}_{1} \times \mathcal{X}_{2} \rightarrow B \times B$ for then restriction to the diagonal in $B \times B$ yields the same formula for the fibred product. Let $p$ (resp. $p_{1}, p_{2}$ ) be the Hilbert polynomial of the fibres of $\mathcal{X}$ (resp. $\left.\mathcal{X}_{1}, \mathcal{X}_{2}\right)$, so $p=p_{1} p_{2}$. Let $\lambda_{\mathcal{X}}(k)=\operatorname{det} \pi_{!}\left(\mathcal{L}^{k}\right)$ (and similarly for $\left.\lambda_{\mathcal{X}_{i}}\right)$. Then by the Künneth formula for $k \gg 0, \lambda_{\mathcal{X}}(k)=\lambda_{\mathcal{X}_{1}}(k)^{p_{2}(k)} \otimes \lambda_{\mathcal{X}_{2}}(k)^{p_{1}(k)}$ so

$$
\lambda_{\text {Hilb }}\left(\mathcal{X}, \mathcal{L}^{r}, k\right)=\lambda_{\text {Hilb }}\left(\mathcal{X}_{1}, \mathcal{L}^{r}, k\right)^{p_{2}(k r) p_{2}(r)} \otimes \lambda_{\text {Hilb }}\left(\mathcal{X}_{2}, \mathcal{L}^{r}, k\right)^{p_{1}(k r) p_{1}(r)} .
$$

Applying (3.5) and taking the leading order term in $k, r$ gives the result.

\section{EXAmples on the unstable locus}

In this section we give examples of a families of polarised manifolds for which the CM line is strictly negative. Of course in light of Theorem 4.3 each fibre in such a family must be unstable. Although it is possible to calculate directly using the definition of the CM line in terms of the $\lambda_{i}$ it is more transparent to restrict to the case when $\pi: \mathcal{X} \rightarrow B$ is smooth and $B$ is an irreducible curve. We will use the first Chern classes of the CM line which, from (2.4), is

$$
c_{1}\left(\lambda_{\mathrm{CM}}\right)=\pi_{*}\left[\mu c_{1}(\mathcal{L})^{n+1}+(n+1) c_{1}\left(K_{\mathcal{X} / B}\right) c_{1}(\mathcal{L})^{n}\right] .
$$

The examples are obtained by blowing up points in the fibres of suitable families $\mathcal{X} \rightarrow B$. Suppose $C \subset \mathcal{X}$ is an irreducible reduced curve dominating $B$ and $\pi: C \rightarrow B$ has degree $d$. Consider the blowup $q: \tilde{\mathcal{X}} \rightarrow \mathcal{X}$ of $\mathcal{X}$ along $C$ with exceptional divisor $E$. It is flat over $B$ and generically the fibre $\tilde{\mathcal{X}}_{b}$ is the fibre $\mathcal{X}_{b}$ blown up at the $d$ points consisting of the intersection with $C$.

Fix a relatively ample line bundle $\mathcal{L} \rightarrow \mathcal{X}$ and consider the $\mathbb{Q}$-line bundle

$$
\mathcal{L}_{\epsilon}=q^{*} \mathcal{L} \otimes \mathcal{O}(-\epsilon E),
$$

on $\tilde{\mathcal{X}}$ which is relatively ample for positive $\epsilon$ sufficiently small. We now compare the $\mathrm{CM}$ lines of $(\mathcal{X}, \mathcal{L})$ and $\left(\tilde{\mathcal{X}}, \mathcal{L}_{\epsilon}\right)$.

Proposition 5.1. Suppose $\pi: \mathcal{X} \rightarrow B$ has relative dimension $n=2$. Then

$$
c_{1}\left(\lambda_{\mathrm{CM}}\left(\tilde{\mathcal{X}}, \mathcal{L}_{\epsilon}\right)\right)=c_{1}\left(\lambda_{\mathrm{CM}}(\mathcal{X}, \mathcal{L})\right)+\epsilon \sigma+O\left(\epsilon^{2}\right)
$$

where

$$
\sigma=\pi_{*}\left(-\frac{d}{a_{0}} c_{1}(\mathcal{L})^{3}+6 c_{1}\left(\left.\mathcal{L}\right|_{C}\right)\right)
$$

Proof. Since $C$ has codimension 2, the relative canonical divisors of $\tilde{\mathcal{X}}$ and $\mathcal{X}$ are related by

Notice that

$$
K_{\tilde{\mathcal{X}} / B}=q^{*} K_{\mathcal{X} / B}+E .
$$

$$
c_{1}\left(q^{*} \mathcal{L}\right)^{2} \cdot c_{1}(E)=q^{*} c_{1}\left(\left.\mathcal{L}\right|_{C}\right)^{2}=0
$$

as $C$ has dimension 1. Similarly $c_{1}(\mathcal{L}) \cdot c_{1}\left(q^{*} K_{\mathcal{X} / B}\right) \cdot c_{1}(E)=0$, and as $\left.\mathcal{O}(E)\right|_{E}$ is the tautological line, $-c_{1}\left(q^{*} \mathcal{L}\right) \cdot c_{1}(E)^{2}=c_{1}\left(\left.\mathcal{L}\right|_{C}\right)$. Thus

$$
\begin{aligned}
c_{1}\left(\mathcal{L}_{\epsilon}\right)^{3} & =q^{*} c_{1}(\mathcal{L})^{3}+O\left(\epsilon^{2}\right) \\
c_{1}\left(K_{\tilde{\mathcal{X}} / B}\right) \cdot c_{1}\left(\mathcal{L}_{\epsilon}\right)^{2} & =\left[c_{1}\left(q^{*} K_{\mathcal{X} / B}\right)+c_{1}(E)\right] \cdot\left[c_{1}\left(q^{*} \mathcal{L}\right)^{2}-2 \epsilon c_{1}\left(q^{*} \mathcal{L}\right) c_{1}(E)+O\left(\epsilon^{2}\right)\right] \\
& =c_{1}\left(K_{\mathcal{X} / B}\right) \cdot c_{1}(\mathcal{L})^{2}+2 \epsilon c_{1}\left(\left.\mathcal{L}\right|_{C}\right)+O\left(\epsilon^{2}\right) .
\end{aligned}
$$


Denote the Hilbert polynomial of the fibres of $\left(\tilde{\mathcal{X}}, \mathcal{L}_{\epsilon}\right)$ by $p_{\epsilon}(k)=a_{0}(\epsilon) k^{2}+a_{1}(\epsilon) k+$ $O\left(k^{0}\right)$, so $p_{0}(k)=p(k)=a_{0} k^{2}+a_{1} k^{1}+O\left(k^{0}\right)$ is the Hilbert polynomial of the fibre of $(\mathcal{X}, \mathcal{L})$. By the Riemann-Roch theorem applied to $\tilde{\mathcal{X}}_{b}$ and $\mathcal{X}_{b}$,

$$
\begin{aligned}
& a_{0}(\epsilon)=\frac{1}{2} \int_{\tilde{\mathcal{X}}_{b}} c_{1}\left(\mathcal{L}_{\epsilon}\right)^{2}=\frac{1}{2} \int_{\tilde{\mathcal{X}}_{b}}\left(c_{1}\left(q^{*} \mathcal{L}\right)-\epsilon c_{1}(E)\right)^{2}=a_{0}+O\left(\epsilon^{2}\right) \\
& a_{1}(\epsilon)=-\frac{1}{2} \int_{\tilde{\mathcal{X}}_{b}} c_{1}\left(K_{\tilde{\mathcal{X}} / B}\right) \cdot c_{1}\left(\mathcal{L}_{\epsilon}\right)=a_{1}-\frac{d \epsilon}{2},
\end{aligned}
$$

so as $\mu=a_{1} / a_{0}$,

$$
\mu_{\epsilon}:=\frac{2 a_{1}(\epsilon)}{a_{0}(\epsilon)}=\mu-\frac{d \epsilon}{a_{0}}+O\left(\epsilon^{2}\right) .
$$

Putting this together, if $\tilde{\pi}=\pi \circ q$ then

$$
\begin{aligned}
c_{1} & \left(\lambda_{\mathrm{CM}}\left(\tilde{\mathcal{X}}, \mathcal{L}_{\epsilon}\right)\right)=\tilde{\pi}_{*}\left[\mu_{\epsilon} c_{1}\left(\mathcal{L}_{\epsilon}\right)^{3}+3 c_{1}\left(K_{\tilde{\mathcal{X}} / B}\right) c_{1}\left(\mathcal{L}_{\epsilon}\right)^{2}\right] \\
& =\pi_{*}\left[\left(\mu-\frac{d \epsilon}{a_{0}}\right) c_{1}(\mathcal{L})^{3}+3\left(c_{1}\left(K_{\mathcal{X} / B}\right) \cdot c_{1}(\mathcal{L})^{2}+2 \epsilon c_{1}(\mathcal{L}) \cdot C\right)+O\left(\epsilon^{2}\right)\right] \\
& =c_{1}\left(\lambda_{\mathrm{CM}}(\mathcal{X}, \mathcal{L})\right)+\epsilon \sigma+O\left(\epsilon^{2}\right)
\end{aligned}
$$

which proves the Proposition.

We will apply this to examples where $\lambda_{\mathrm{CM}}(\mathcal{X}, \mathcal{L})$ has zero degree and $\sigma<0$, obtaining examples of families of (smooth) polarised varieties for which the $\mathrm{CM}$ line has strictly negative degree.

Example 5.3. Let $B=\mathbb{P}^{1}$ and consider the vector bundle

$$
\mathcal{E}=\mathcal{O}_{\mathbb{P}^{1}}(2) \oplus \mathcal{O}_{\mathbb{P}^{1}}(-1) \oplus \mathcal{O}_{\mathbb{P}^{1}}(-1) .
$$

We let $\mathcal{X}=\mathbb{P}(\mathcal{E})$ (the space of lines in $\mathcal{E}$ ) and $q: \tilde{\mathcal{X}} \rightarrow \mathcal{X}$ be the blowup along the curve $C=\mathbb{P}\left(\mathcal{O}_{\mathbb{P}^{1}}(2)\right) \cong B$. Set $\mathcal{L}=\mathcal{O}_{\mathbb{P}(\mathcal{E})}(1)$ which is relatively ample and $\mathcal{L}_{\epsilon}=q^{*} \mathcal{L} \otimes \mathcal{O}(-\epsilon E)$ where $E$ is the exceptional set of the blowup.

As $c_{1}(\mathcal{E})=0$ we have for $k \gg 0, c_{1}\left(\pi_{*} \mathcal{L}^{k}\right)=c_{1}\left(S^{k} \mathcal{E}^{*}\right)=0$. Hence $c_{1}\left(\lambda_{i}\right)=0$ for all $i$, which implies $c_{1}\left(\lambda_{\mathrm{CM}}(\mathcal{X}, \mathcal{L})\right)=0$. Moreover, $\pi_{*}\left(c_{1}(\mathcal{L})^{3}\right)=0$ and $c_{1}\left(\left.\mathcal{L}\right|_{C}\right)=$ -2 so $\sigma=-12$. Thus by Proposition [5.1]

$$
c_{1}\left(\lambda_{\mathrm{CM}}\left(\tilde{\mathcal{X}}, \mathcal{L}_{\epsilon}\right)\right)<0 \quad \text { for } 0<\epsilon \ll 1 .
$$

Notice that the fibres of $\tilde{\mathcal{X}}$ consist of $\mathbb{P}^{2}$ blown up at a single point, which is known to be unstable with respect to any polarisation (13], Example 5.27).

Remark 5.4. This previous example is similar to one due to Morrison [3] showing that the Cornalba-Harris line $\lambda_{\mathrm{CH}}$ (3.3) is negative on a family of Steiner surfaces. We remark that in fact $\lambda_{\mathrm{CH}}\left(\tilde{\mathcal{X}}, \mathcal{L}_{\epsilon}^{r}\right)$ is negative for fixed $0 \leq \epsilon \ll 1$ and $r \gg 0$.

Example 5.5. In Example 5.3 we could instead have let $C=\mathbb{P}\left(\mathcal{O}_{\mathbb{P}^{1}}(-1)\right)$. Then the fibres of $\tilde{\mathcal{X}}$ are still all unstable with respect to any polarisation. However $c_{1}\left(\left.\mathcal{L}\right|_{C}\right)=1$ and $\sigma=6$ so $c_{1}\left(\lambda_{\mathrm{CM}}\left(\tilde{\mathcal{X}}, \mathcal{L}_{\epsilon}\right)\right)>0$. Thus the CM line need not always be strictly negative on the unstable locus. Notice that the Hilbert polynomial of the fibres in this example are the same as in Example 5.3 Thus there are fixed Hilbert schemes on which the CM line is neither strictly positive nor strictly negative on the unstable locus. 
Example 5.6. The fibres of the family in Example 5.3 have non-trivial infinitesimal automorphisms. However, this is not necessary for the CM line to be negative. To see this consider the family $\left(\mathcal{X}, \mathcal{L}_{\epsilon}\right)$ in Example 5.3 and fix $0<\epsilon \ll 1$ so $\lambda_{\mathrm{CM}}\left(\tilde{\mathcal{X}}, \mathcal{L}_{\epsilon}\right)$ is strictly negative. Pick sections $C_{1}, C_{2}, C_{3}$ of $\pi$ so that $C, C_{1}, C_{2}, C_{3}$ meet the generic fibre of $\mathcal{X}$ in 4 generic points. Again let $\tilde{\mathcal{X}}$ be the blowup of $\mathcal{X}$ along $C$ and denote their proper transforms of $C_{1}, C_{2}, C_{3}$ in $\tilde{\mathcal{X}}$ also by $C_{i}$ (so $C_{i}$ is disjoint from $E)$.

Let $p: \mathcal{Y} \rightarrow \tilde{\mathcal{X}}$ along $\cup C_{i}$, with exceptional set $E^{\prime}$. For $0<\epsilon^{\prime} \ll \epsilon$ the line $\mathcal{L}^{\prime}=p^{*}(\mathcal{L} \otimes \mathcal{O}(-\epsilon E)) \otimes \mathcal{O}\left(-\epsilon^{\prime} E^{\prime}\right)$ is relatively ample, and by continuity $\lambda_{\mathrm{CM}}\left(\mathcal{Y}, \mathcal{L}^{\prime}\right)$ is also strictly negative for $0<\epsilon^{\prime} \ll \epsilon$.

Notice that a fibre of $\mathcal{Y}$ is $\mathbb{P}^{2}$ blown up at 4 distinct points, which do not all lie on a line, and thus has discrete automorphism group. These manifolds are known to be unstable with respect to the polarisations considered here (13], Example 5.30)

Example 5.7. Suppose $\left(\mathcal{X}_{1}, \mathcal{L}_{1}\right)$ (resp. $\left.\left(\mathcal{X}_{2}, \mathcal{L}_{2}\right)\right)$ are families over the same curve $B$ chosen so that the associated CM line is strictly negative (resp. non-positive). Set $\mathcal{X}=\mathcal{X}_{1} \times_{B} \mathcal{X}_{2}$ with projections $q_{i}: \mathcal{X} \rightarrow \mathcal{X}_{1}$ and $\mathcal{L}=q_{1}^{*} \mathcal{L}_{1} \otimes q_{2}^{*} \mathcal{L}_{2}$. Then Corollary 4.7 implies then the CM line of $(\mathcal{X}, \mathcal{L})$ will have strictly negative degree. This gives the existence of families of manifolds of arbitrary dimension for which the $\mathrm{CM}$ line is strictly negative. Notice that since the fibres of $\mathcal{X}_{1}$ are necessarily unstable, the fibres of $\mathcal{X}$ will be as well.

Remark 5.8. It is interesting to note that the idea above can, in principle at least, be used to show that $\lambda_{\mathrm{CM}}$ is ample. Still with $n=2$, suppose that $\sigma<0$ and

- Some fibre $\left(\mathcal{X}_{b}, \mathcal{L}_{b}\right)$ of $(\mathcal{X}, \mathcal{L})$ is smooth, has a discrete automorphism group and $c_{1}\left(\mathcal{L}_{b}\right)$ admits a constant scalar curvature Kähler ( $\left.\operatorname{cscK}\right)$ metric

- The curve $C$ meets $\mathcal{X}_{b}$ in $d$ distinct points.

Arrezo-Pacard [1] show that under these assumption the blowup of $X_{b}$ at the points $C . X_{b}$ admits a cscK metric in classes which make the exceptional set sufficiently small. Thus the fibre of $\left(\mathcal{X}, \mathcal{L}_{\epsilon}\right)$ over $b$ admits a cscK metric. As mentioned previously, a result of Donaldson [4 then implies that this fibre is asymptotically Hilbert semistable and so from (4.3),$c_{1}\left(\lambda_{\mathrm{CM}}\left(\tilde{\mathcal{X}}, \mathcal{L}_{\epsilon}\right) \geq 0\right.$ for $0<\epsilon \ll 1$. By Proposition 5.1 the assumption that $\sigma<0$ then implies that $c_{1}\left(\lambda_{\mathrm{CM}}(\mathcal{X}, \mathcal{L})\right)>0$. Unfortunately, we do not know of a specific example where this can be applied, but wonder nevertheless if this idea could be used to show ampleness of the CM line in some cases not covered by Fujiki-Schumacher's result (described in Remark 4.5).

\section{REFERENCES}

[1] C. Arezzo and F. Pacard. Blowing up and desingularizing constant scalar curvature Kähler manifolds, arXiv:math.DG/0411522.

[2] T. Aubin. Équations du type Monge-Ampère sur les variétés kähleriennes compactes. $C$. $R$. Acad. Sci. Paris Sér. A-B, 283(3):Aiii, A119-A121, 1976.

[3] M. Cornalba and J. Harris. Divisor classes associated to families of stable varieties, with applications to the moduli space of curves. Ann. Sci. École Norm. Sup. (4), 21(3):455-475, 1988.

[4] S. K. Donaldson. Scalar curvature and projective embeddings. I. J. Differential Geom., 59(3):479-522, 2001.

[5] S. K. Donaldson. Scalar curvature and stability of toric varieties. J. Differential Geom., 62(2):289-349, 2002.

[6] J. Fine. Fibrations with constant scalar curvature Kahler metrics and the CM-line bundle, arXiv:math.DG/0510075. 
[7] A. Fujiki and G. Schumacher. The moduli space of extremal compact Kähler manifolds and generalized Weil-Petersson metrics. Publ. Res. Inst. Math. Sci., 26(1):101-183, 1990.

[8] D. Gieseker. Global moduli for surfaces of general type. Invent. Math., 43(3):233-282, 1977.

[9] J. Harris and I. Morrison. Moduli of curves, volume 187 of Graduate Texts in Mathematics. Springer-Verlag, New York, 1998.

[10] F. F. Knudsen and D. Mumford. The projectivity of the moduli space of stable curves. I. Preliminaries on "det" and "Div". Math. Scand., 39(1):19-55, 1976.

[11] D. Mumford, J. Fogarty, and F. Kirwan. Geometric invariant theory. Springer-Verlag, Berlin, third edition, 1994.

[12] S. T. Paul and G. Tian. CM stability and the generalized Futaki invariant I. preprint.

[13] J. Ross and R. P. Thomas. An obstruction to the existence of constant scalar curvature Kähler metrics. Journal of Differential Geometry 72, 429-466, 2006, 2005, arXiv:math.DG/0412518.

[14] G. Tian. The K-energy on hypersurfaces and stability. Comm. Anal. Geom., 2(2):239-265, 1994.

[15] G. Tian. Canonical metrics in Kähler geometry. Birkhäuser Verlag, Basel, 2000. Notes taken by Meike Akveld.

[16] E. Viehweg. Weak positivity and the additivity of the Kodaira dimension for certain fibre spaces. In Algebraic varieties and analytic varieties (Tokyo, 1981), volume 1 of Adv. Stud. Pure Math., pages 329-353. North-Holland, Amsterdam, 1983.

[17] S. T. Yau. On the Ricci curvature of a compact Kähler manifold and the complex MongeAmpère equation. I. Comm. Pure Appl. Math., 31(3):339-411, 1978.

joel.fine@imperial.ac.uk

Department of Mathematics, Imperial College, London SW7 2AZ. UK.

jaross@math. columbia.edu

Department of Mathematics, Columbia University, New York, NY 10027. USA. 